\title{
The promised land
}

Eytan M. Stein

Department of Medicine, Memorial Sloan Kettering Cancer Center, New York, NY, USA

E-mail: EYTAN M. STEIN - steine@mskcc.org

doi:10.3324/haematol.2020.276428

I 2021, most, if not all, presentations and publications about the treatment of acute myeloid leukemia (AML) start biblically: hematologists spent 40 years in the desert of "7+3" and in 2017 reached a promised land flowing with Food and Drug Administration approvals. One of the first approvals addressed the vexing problem of FLT3mutant AML. While the FLT3-internal tandem duplication (ITD) was discovered over 25 years ago, the success of randomized clinical studies targeting the mutant FLT-3 protein only occurred years later with the RATIFY trial. ${ }^{1}$ In this trial, the combination of the multikinase inhibitor midostaurin with induction chemotherapy led to a statistically significant improvement in the 4-year overall survival of patients with newly diagnosed FLT3-mutant AML. ${ }^{2}$ The approval of single-agent gilteritinib, a second-generation FLT-3 inhibitor for patients with relapsed and refractory AML, based on the randomized phase III ADMIRAL trial, consolidated the role of FLT-3 inhibitors in the treatment of patients with AML. ${ }^{3}$

But while gilteritinib was being approved, a second FLT3 inhibitor, quizartinib, was axed by the Oncologic Drugs Advisory Committee (ODAC). Quizartinib, a potent inhibitor of FLT3-ITD but not FLT3-tyrosine kinase domain (TKD) mutations, was studied in a randomized phase III trial, for relapsed and refractory AML. Despite demonstrating a statistically significant overall survival benefit in the company-sponsored analysis of the clinical trial, concerns about dropouts in the standard treatment arm led to a negative vote against quizartinib at an ODAC meeting. Despite this, quizartinib was approved in Japan and is now a standard-of-care therapy in that country.

Building on previous work showing the relative benefit of treating patients with the combination of sorafenib, a weak FLT-3 inhibitor, with azacitidine, in this issue of Haematologica, Swaminathan and colleagues ${ }^{4}$ report the outcomes of patients with either newly diagnosed or relapsed FLT3-ITD AML treated with the combination of azacitidine or low-dose cytarabine with quizartinib. Perhaps the most impressive outcome in this trial is the high rate of composite complete response of $87 \%$ with quizartinib/azacitidine and $74 \%$ with quizartinib/lowdose cytarabine. These rates of remission are certainly encouraging, although definitive results will need to wait for the time of a randomized, ideally placebo-controlled, study. As a cautionary note, the randomized phase III LACEWING study, in which patients were assigned to treatment with the two-drug combination of gilteritinib/azacitidine or azacitidine monotherapy, failed to meet its primary endpoint of an overall survival benefit in favor of the former despite encouraging results of the "doublet" in a phase II clinical trial.

Concurrent with the development of treatments targeting FLT-3, there has been a dramatic improvement in the outcomes of adults who are not considered good candidates for induction chemotherapy with the use of azacitidine and venetoclax. FLT3-mutant patients appear to do as well with azacytidine/venetoclax as patients without FLT3 mutations, at least when it comes to response. How then, should we think about the doublet of azacitidine/quizartinib? The field of leukemia research is now moving past doublets, and Swaminathan and colleagues have set a firm foundation for thinking about triplets of azacitidine/venetoclax/quizartinib or sequential treatments with azacitidine/venetoclax followed by azacitidine/quizartinib. Studies on these combinations will take time to perform but are worthwhile and will continue to improve the lives of all of our patients with FLT3-mutant AML.

\section{Disclosures}

No conflicts of interest to disclose.

\section{References}

1. Nakao M, Yokota S, Iwai T, et al. Internal tandem duplication of the flt3 gene found in acute myeloid leukemia. Leukemia. 1996;10(12):1911-1918.

2. Stone RM, Mandrekar SJ, Sanford BL, et al. Midostaurin plus chemotherapy for acute myeloid leukemia with a FLT3 mutation. N Engl J Med. 2017;377(5):454-464.

3. Perl AE, Martinelli G, Cortes JE, et al. Gilteritinib or chemotherapy for relapsed or refractory FLT3-mutated AML. N Engl J Med. 2019;381(18):1728-1740.

4. Swaminathan M, Kantarjian HM, Levis M, et al. A phase I/II study of the combination of quizartinib with azacitidine or low-dose cytarabine for the treatment of patients with acute myeloid leukemia and myelodysplastic syndrome. Haematologica. 2021;106(8):2121-2130 